\title{
Influence de la distribution d'un supplément solide sur la physiologie digestive du veau de boucherie
}

\author{
JH Burton ${ }^{1}, \mathrm{R}$ Toullec $^{1}, \mathrm{P}$ Guilloteau ${ }^{1}, \mathrm{~T}_{\text {Corring }}{ }^{2}, \mathrm{JA}$ Chayvialle $^{3}$ \\ $C^{\prime}$ Bernard $^{3}, P$ Barré ${ }^{4}$ \\ 1 INRA, laboratoire du jeune ruminant, 65, rue de Saint-Brieuc, 35042 Rennes Cedex; \\ 2 INRA, laboratoire d'écologie et de physiologie du système digestif, \\ 78352 Jouy-en-Josas, Cedex; \\ 3 INSERM, U45, hôpital E-Herriot, 69374 Lyon Cedex; \\ ${ }^{4} U C A A B, B P 19,02400$ Château-Thierry, France
}

\begin{abstract}
Summary - Effect of giving a solid supplement on the digestive physiology of the preruminant calf. Feeding small amounts of a fibrous pelleted concentrate to milk-fed veal calves reduced the number of hairballs in the rumen and increased the weight of the forestomachs and the specific activities of trypsin, chymotrypsin and lipase.
\end{abstract}

Le veau de boucherie reçoit traditionnellement un régime exclusivement lacté. Le poids à l'abattage ayant considérablement augmenté au cours des dernières années, il pourrait être intéressant de distribuer de petites quantités de supplément solide riche en fibres, afin, notamment, d'améliorer le bien-être des animaux. L'objet du présent travail était d'étudier les conséquences d'une telle pratique sur la rétention azotée et le fonctionnement du tube digestif.

Matériel et méthodes - Dans un premier essai, 24 veaux mâles Holstein, achetés à l'âge d'environ $8 \mathrm{j}$, reçoivent un régime lacté intensif pendant $118 \mathrm{j}$ (216 kg de matière sèche (MS) d'aliment d'allaitement contenant $24 \%$ de matières azotées (MA) et $22 \%$ de lipides). Douze d'entre eux disposent en plus d'un aliment granulé (Maturex ${ }^{\mathrm{P}}$, UCAAB), à base de paille et de céréales $(14,5 \mathrm{~kg}$ de MS contenant $11 \%$ de MA, $14 \%$ de cellulose brute, $4 \%$ de matières minérales et $2 \%$ de lipides). La quantité de MS distribuée par jour est de $45,90,136$ et $181 \mathrm{~g}$ de 2 à 28 ,
29 à 49,50 à 70 et au-delà de $70 \mathrm{j}$, respectivement. Les veaux sont élevés sur caillebotis. Des mesures de bilans digestifs (matière organique, $\mathrm{N}$, lipides, $\mathrm{Ca}$ et $\mathrm{P}$ ) ont lieu en semaine $4,8,12$ et 16 . Dans un second essai, 2 veaux sont munis d'une poche abomasale innervée. Le lait est distribué seul pendant 4 semaines, puis avec le supplément solide pendant 4 autres semaines. L'un des veaux, demeuré fonctionnel à l'issue de ces 2 périodes, est sevré et reçoit du foin et de l'aliment concentré pendant 4 semaines après le sevrage.

Résultats et discussion - Dans le premier essai, la digestibilité apparente, la rétention azotée, la concentration plasmatique d'urée, le gain de poids vif, ainsi que le poids de la carcasse, du pancréas, de la caillette, de l'intestin grêle, du colon et du contenu de ces 3 derniers organes sont identiques avec les 2 régimes. En revanche, le poids des préestomacs et celui du cæcum sont plus élevés avec le supplément solide ( $1364 \pm 55$ et $364 \pm 101$ au lieu de $1082 \pm 39$ et $232 \pm 16 ; P \leq 0,01)$. II 
en est de même pour le contenu des préestomacs ( $3690 \pm 526$ au lieu de $1762 \pm 397)$. Le rendement commercial est donc légèrement abaissé $\langle 60,6 \pm 0,3$ au lieu de $61,5 \pm 0,3$; $P<0,05)$. Le nombre de veaux ayant des aegagropiles dans le réticulorumen diminue (2 au lieu de 10), ainsi que le nombre d'aegagropiles, chez ces animaux $(6,6 \pm 1,7$ et 15,5 $\pm 4,3)$. Aucune caillette ne semble comporter d'ulcères dans le lot supplémenté, tandis que 3 d'entre elles présentent 1 ou 2 ulcères cicatrisés dans le lot témoin. Ces résultats ne s'accordent pas avec ceux de Wensing et al (1986), qui ont observé une augmentation de la fréquence des ulcères avec différents suppléments solides. Les activités enzymatiques de la caillette ne sont pas significativement affectées, bien qu'elles tendent à être réduites pour la chymosine et accrues pour la pepsine dans le lot supplémenté (tableau I). En revanche, au niveau du pancréas, les activités spécifiques de la trypsine, de la chymotrypsine et de la lipase sont plus fortes dans le lot supplémenté; toutefois, seule la différence concernant la

Tableau I. Activités enzymatiques dans la caillette (unités/g de muqueuse) et le pancréas (unités/mg de protéines) (moyennes \pm écarts types des moyennes).

\begin{tabular}{|c|c|c|}
\hline Lot & Témoin & Supplémenté \\
\hline Lysozyme & $1206 \pm 118$ & $1162 \pm 80$ \\
\hline Chymosine & $6344 \pm 608$ & $5035 \pm 605$ \\
\hline Pepsine & $1130 \pm 160$ & $1310 \pm 137$ \\
\hline Trypsine* & $0,81 \pm 0,03$ & $1,15 \pm 0,08$ \\
\hline Chymotrypsine & $7,00 \pm 0,46$ & $10,06 \pm 0,41$ \\
\hline Lipase* & $4,46 \pm 0,13$ & $6,96 \pm 0,38$ \\
\hline Amylase & $51,2 \pm 5,40$ & $49,9 \pm 3,80$ \\
\hline
\end{tabular}

"Différence significative entre lots $(P \leq 0,01)$. lipase reste significative pour les activités totales par veau. La concentration duodénale de motiline immunoréactive est plus élevée dans le lot supplémenté (824 $\pm 193 \mathrm{ng} / \mathrm{g}$ de tissu au lieu de $435 \pm 79 ; P<0,10)$. En revanche, les taux plasmatiques et antraux, duodénaux ou pancréatiques de gastrine CCK, VIP, GIP, PP, sécrétine et somatostatine ne sont pas modifiés. Dans le second essai, la quantité d'ions $\mathrm{H}^{+}$ sécrétés augmente lorsque le supplément solide est introduit dans la ration (de $53 \pm 7 \mathrm{méq} / 100 \mathrm{~kg}$ de poids vif à $68 \pm 9$ et de $117 \pm 17$ à $142 \pm 7$ chez le $1^{\text {er }}$ et le $2^{e}$ veau), mais aucune des différences n'est significative. Cette augmentation reste faible comparativement à celle qui est observée après le sevrage chez le $1^{\text {or }}$ veau $(262 \pm$ 20 méq $/ 100 \mathrm{~kg}$ de poids vif; $P \leq 0,05$ par rapport aux 2 périodes précédentes).

En conclusion, la distribution du supplément solide influence le fonctionnement du tube digestif. Toutefois, les effets sont modérés par rapport à ceux observés en passant à un régime exclusivement solide (Guilloteau et al, 1985) et n'empêchent pas les veaux de demeurer essentiellement des préruminants.

Remerciements - Ce travail a bénéficié du soutien de la région de Bretagne et de la direction générale de l'Alimentation du ministère de l'Agriculture.

Guilloteau P, Corring T, Toullec R, Guilhermet R (1985) Reprod Nutr Dév 25, 481-493

Wensing T, Breukink HJ, Van Dijk S (1986) Res Vet Sci 10, 195-202 